\title{
Transatlantica
}

Revue d'études américaines. American Studies Journal

\section{Exposition Mitch Epstein : American Power}

Du 4 mai au 24 juillet 2011, Fondation Henri Cartier-Bresson

\section{Géraldine Fasentieux}

\section{(2) OpenEdition}

Journals

Édition électronique

URL : http://journals.openedition.org/transatlantica/5373

DOI : $10.4000 /$ transatlantica. 5373

ISSN : $1765-2766$

Éditeur

AFEA

Référence électronique

Géraldine Fasentieux, «Exposition Mitch Epstein : American Power », Transatlantica [En ligne], 1| 2011, mis en ligne le 03 janvier 2012, consulté le 29 avril 2021. URL : http://journals.openedition.org/

transatlantica/5373 ; DOI : https://doi.org/10.4000/transatlantica.5373

Ce document a été généré automatiquement le 29 avril 2021

\section{cc)}

Transatlantica - Revue d'études américaines est mis à disposition selon les termes de la licence Creative Commons Attribution - Pas d'Utilisation Commerciale - Pas de Modification 4.0 International. 


\section{Exposition Mitch Epstein : American Power}

Du 4 mai au 24 juillet 2011, Fondation Henri Cartier-Bresson

\section{Géraldine Fasentieux}

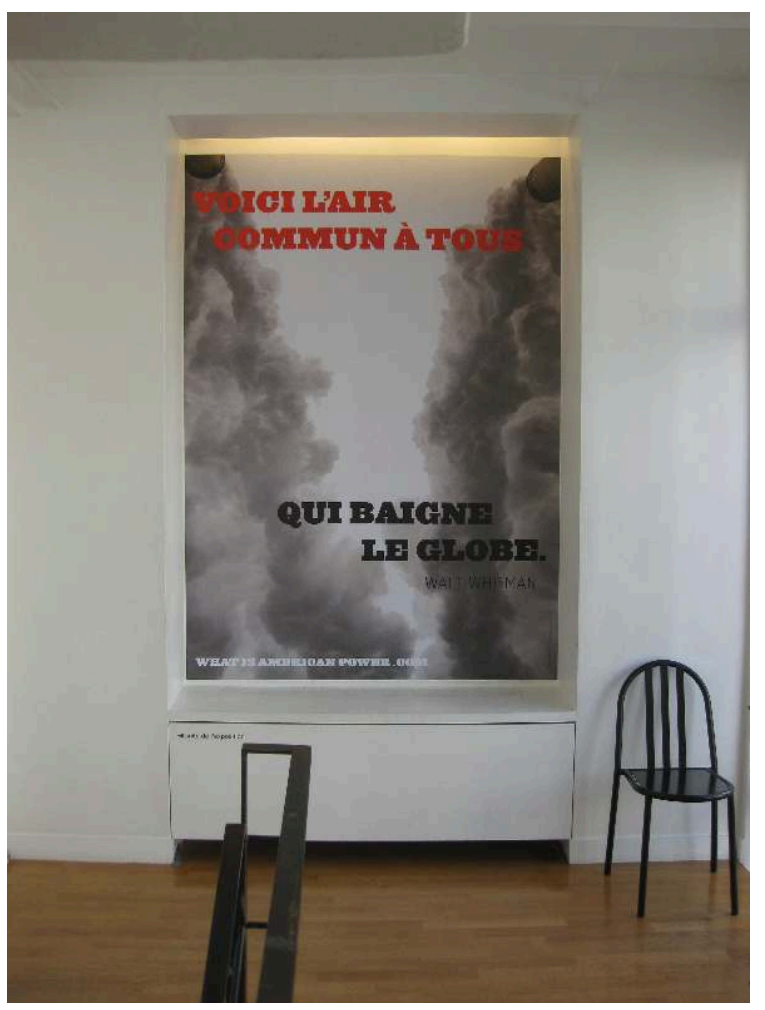

Photo reproduite avec l'aimable autorisation de la Fondation HCB

En 2003, le documentariste américain Mitch Epstein reçoit une commande du New York Times, qui lui demande de photographier la destruction de Cheshire, un village d'environ 300 habitants dans l'Ohio. Le village, contaminé par la centrale thermique voisine doit être abandonné et rayé de la carte en raison d'une catastrophe écologique. 
L'AEP (American Electric Power), propriétaire de la centrale, dédommage les habitants afin que ceux-ci déménagent et s'engagent à ne jamais les poursuivre en cas de problèmes de santé liés aux produits toxiques relâchés dans la nature par la centrale de l'AEP, ni à se plaindre auprès des médias. Pour le photographe, « la société s'achetait un avenir à l'abri de poursuites judiciaires. » Il assiste, incrédule, à ce jeu de massacre. « La destruction était si rapide et si facile que je devais me répéter que j'avais sous les yeux des maisons réelles, pas des jouets en carton. [...] De retour à New York, il me fut impossible d'oublier Cheshire» raconte-t-il. C'est un visage, celui d'une irréductible octogénaire, Beulah Hern, dite « Boots », qui laisse sur le photographe «l'empreinte la plus profonde». Refusant de vendre sa maison, elle se protège par des caméras de surveillance et son arme rangée dans la poche latérale de son fauteuil relax.

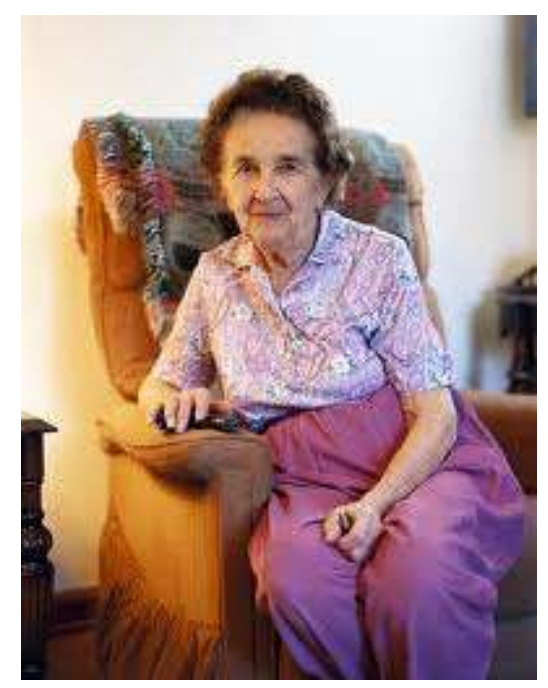

Beulah Hern, dite « Boots », Cheshire, Ohio, 2004. Droits réservés.

2 Frappé par la situation dramatique des résidents qui voient leur maison rasée en quelques minutes, Mitch Epstein décide alors d'enquêter sur la production et la consommation d'énergie aux États-Unis, afin de tenter de comprendre leur influence sur le paysage et la société américaine. C'est ainsi que naît le projet American Power, titre de l'exposition présentée à la fondation Henri Cartier-Bresson du 4 mai au 24 juillet 2011, et aussi du catalogue éponyme de 63 photographies prises entre 2003 et 2008 dans vingt-cinq États, qui montrent les sites de production énergétique ou leurs environs, et surtout, leur impact sur le paysage américain. «Je voulais photographier la relation qui existe entre la société américaine et le paysage américain » écrit Epstein. Comme son titre l'indique, cette série donne à voir la relation entre énergie et pouvoir, afin d'interroger, selon les mots du photographe, "la mainmise de l'homme sur la nature, et sa conquête à n'importe quel prix ». Ce travail lui vaut d'obtenir le prix Pictet, qui récompense les messages d'envergure environnementale globale. Ce que l'on peut voir comme l'essence du capitalisme irresponsable qui conduit à des catastrophes nucléaires comme Tchernobyl ou Fukushima est ainsi présenté sur les cimaises de la fondation. L'accrochage reprend l'exact déroulé de l'ouvrage. 


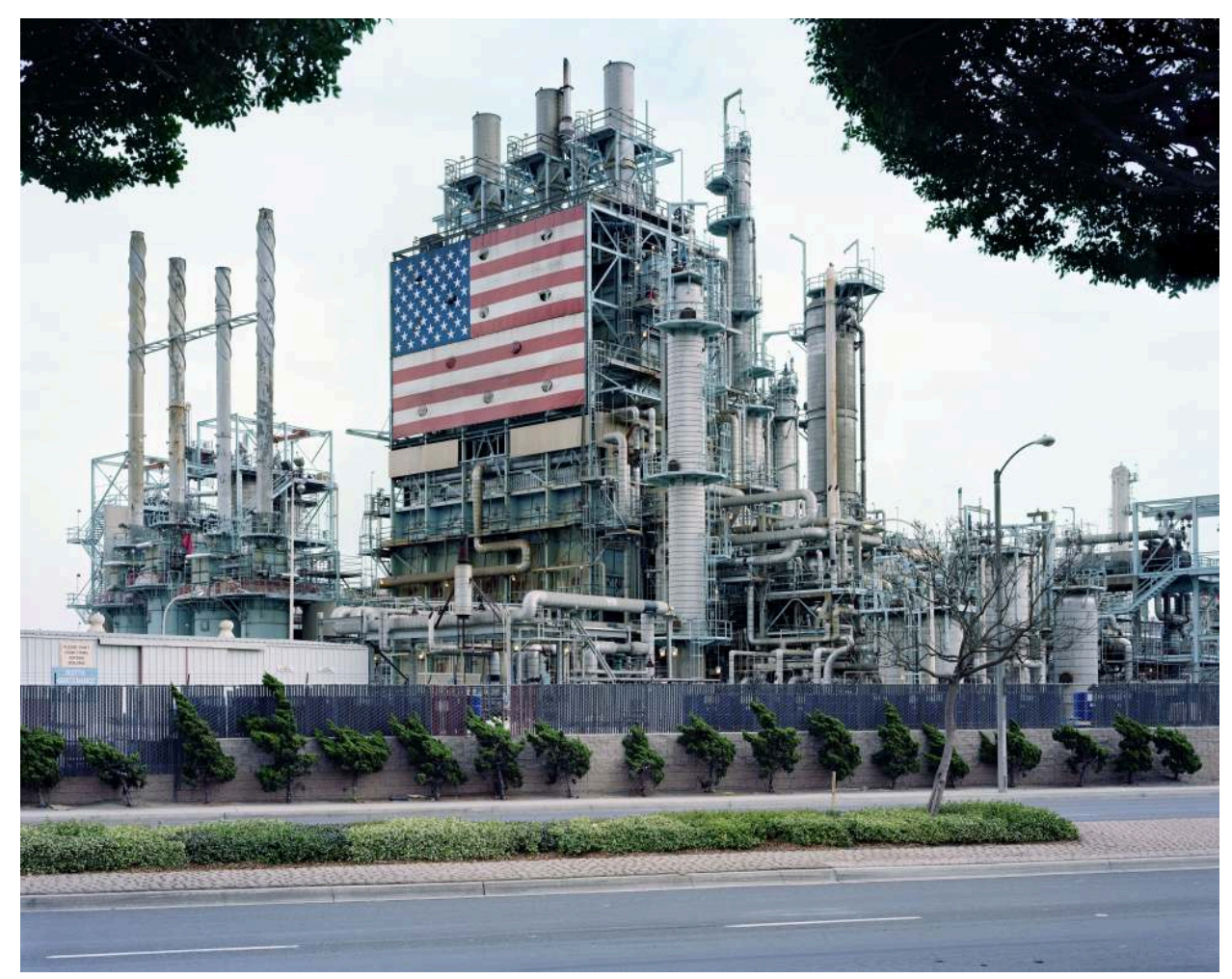

Raffinerie BP à Carson, Californie, 2007. COPYRIGHT Black River Productions, Ltd. / Mitch Epstein. Photo reproduite avec l'aimable autorisation de la Galerie Thomas Zander, Cologne.

3 Pour effectuer ce travail, le photographe doit faire face à de nombreuses tentatives de dissuasion, comme si montrer une certaine réalité peu glorieuse constituait une menace.

tout au long de mon travail sur American Power, j'ai souffert d'un harcèlement systématique. À maintes reprises, les forces de police m'ont chassé alors que je n'avais commis aucun délit. Avec pour résultat qu'entre 2003 et 2008 - une période qui a coïncidé avec l'ère Bush - quasiment partout où j'allais aux États-Unis pour travailler, j'avais la peur au ventre. L'explication, c'est que mes intentions contrecarraient les intérêts de l'industrie, qui étaient soutenus par le département de la Sécurité intérieure. Je voulais rendre le dossier de l'énergie plus transparent, tandis que les grosses sociétés qui produisaient l'énergie et leurs interlocuteurs gouvernementaux s'enveloppaient d'une atmosphère de secret. 


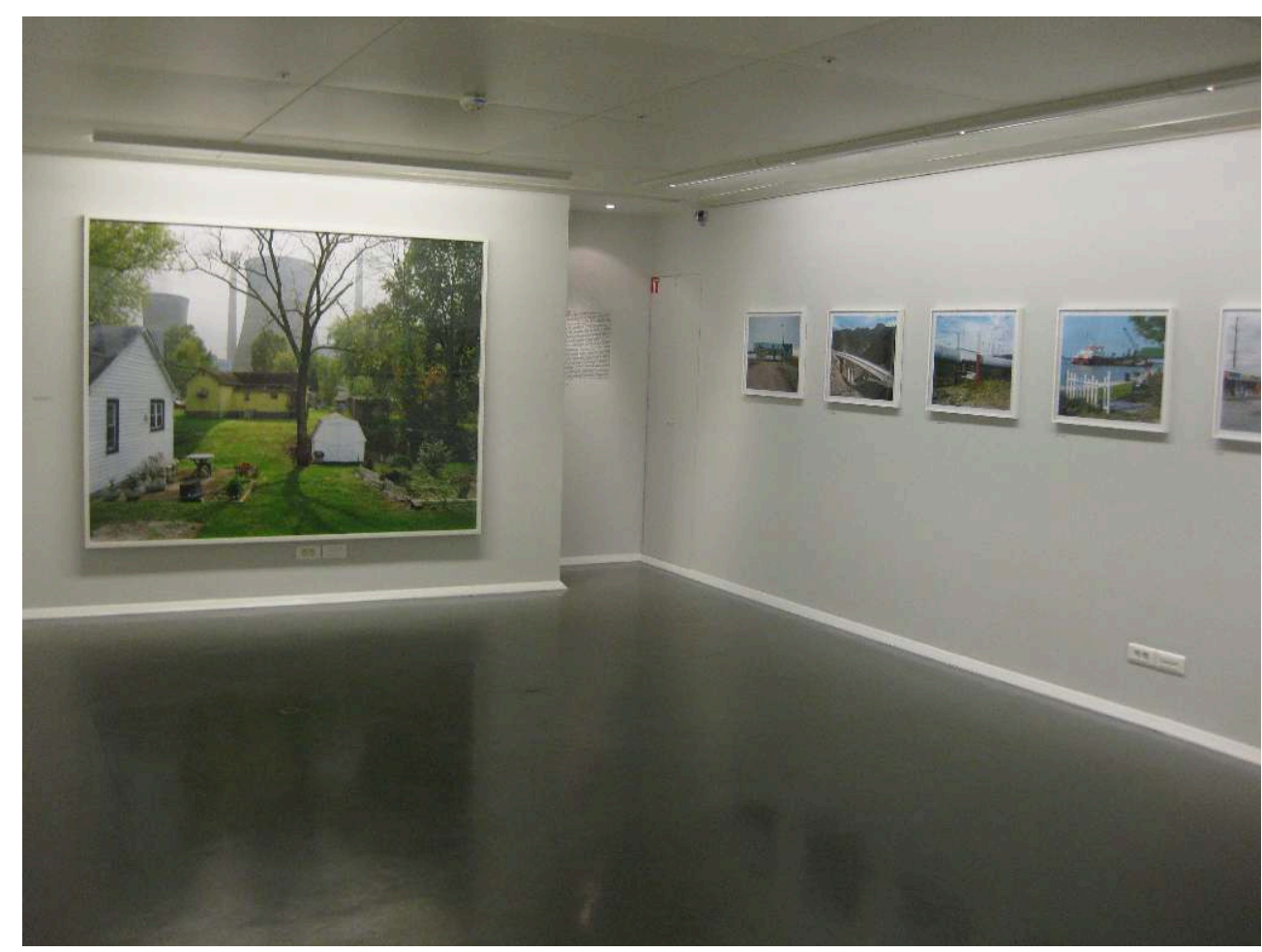

Certes, les images qui font froid dans le dos sont nombreuses, car la quiétude qui en émane est rompue par une menace sourde, une présence devenue tout aussi familière que le paysage naturel. Les lieux de production d'énergie font partie d'un décor quotidien pour certains, un background que l'on semble presque oublier. C'est ainsi que l'on peut voir les gigantesques cheminées servir de toile de fond à l'arrière d'un jardin joliment entretenu, et où, d'après la propriétaire, le photographe aura « la plus belle vue sur les tours de refroidissement». Les clichés montrent les centrales dans un paysage naturel où l'homme continue à vivre comme si de rien n'était. Des centrales thermiques ou nucléaires côtoient le terrain de foot d'un lycée en Virginie, ou encore des plages où les gens se baignent ou pêchent tranquillement en Californie ou en Floride. Les éoliennes qui font désormais partie du décor se veulent plus rassurantes. Ce qui est frappant, c'est que les hommes semblent composer de façon naturelle avec ce décor insolite, voire effrayant, et surtout dangereux, dont les conséquences néfastes peuvent parfois être irréversibles. 


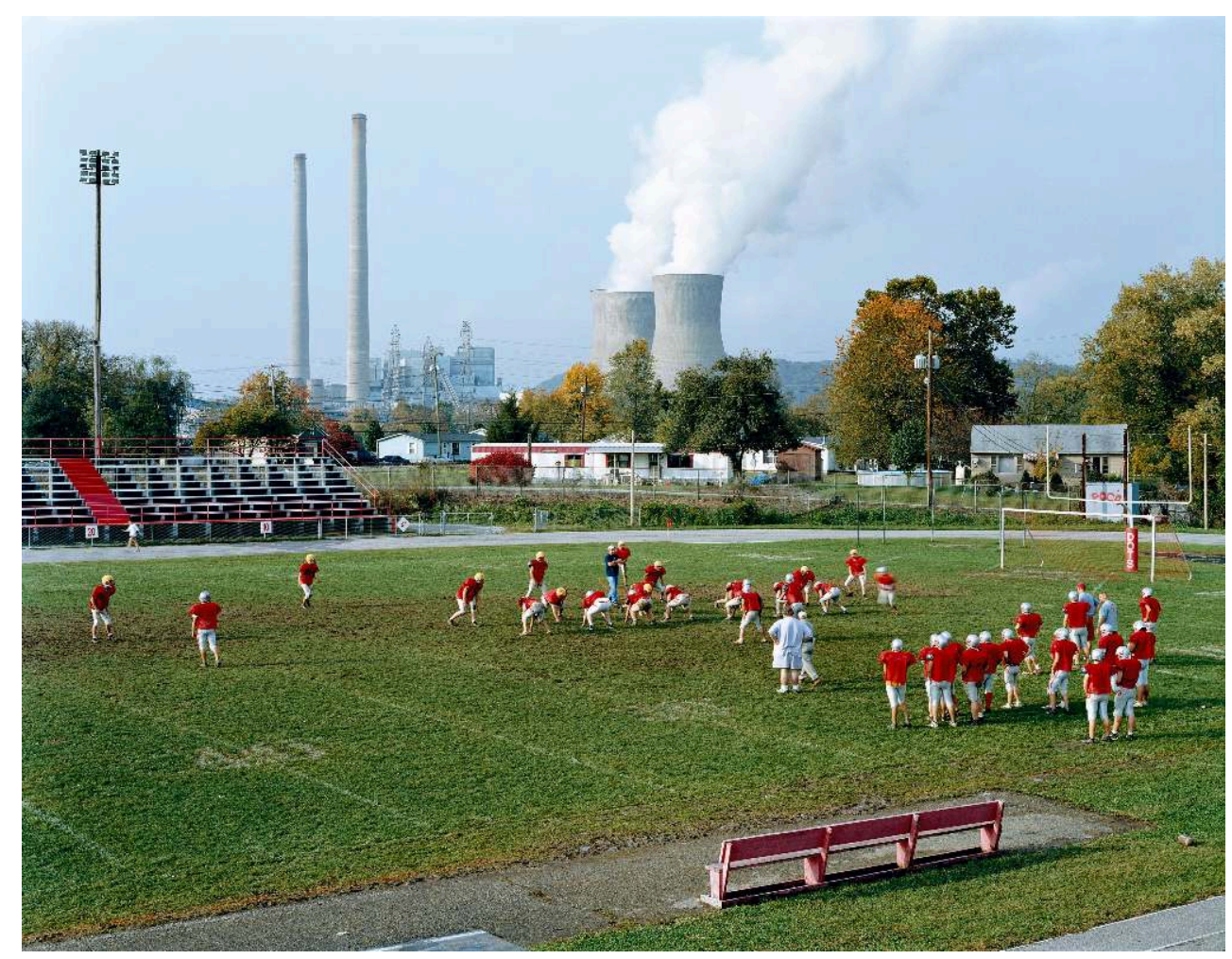

Lycée de Poca et centrale thermique d'Amos, Virginie Occidentale, 2004. COPYRIGHT Black River Productions, Ltd. / Mitch Epstein. Photo reproduite avec l'aimable autorisation de la Galerie Thomas Zander, Cologne

5 A travers l'objectif de l'appareil photo, les images apparaissent d'une beauté monumentale. En regardant les somptueux panoramas dévastés, le spectateur prend conscience de la transformation et de la dégradation du paysage qui découlent de la production d'énergie. Ses découvertes des dégâts que subissent les paysages américains incitent Mitch Epstein à « réévaluer à la fois [son] sens de la légitimité et l'idéologie américaine de la Destinée manifeste ».

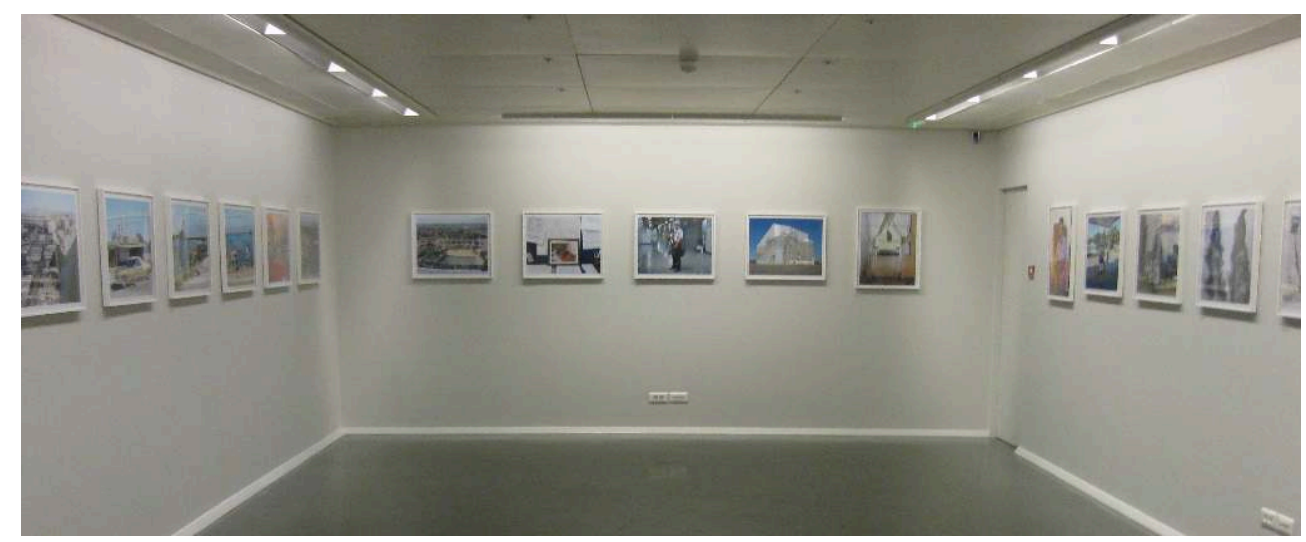

Photo reproduite avec l'aimable autorisation de la Fondation HCB

6 Chacune des images présentées est un choc visuel, qui provoque un sentiment d'écœurement devant la cupidité de l'industrie et son indifférence à l'égard de l'environnement. Parce que le rêve américain de confort matériel a fini «par exiger plus d'énergie que le pays n'en pouvait fournir », Mitch Epstein invite le visiteur à 
« tenir compte de [ses] devoirs envers la nature et envers les autres hommes, et pas seulement de [ses] droits individuels.»

BIBLIOGRAPHIE

EPSTEIN, Mitch, American Power, Göttingen, Steidl, 2011.

INDEX

Thèmes : Trans'Arts 